\title{
ARTíCULO
}

\section{Permanencia en la universidad: la importancia de un buen comienzo}

\author{
María Esteban García ${ }^{\mathrm{a}, *}$, Ana Belén Bernardo Gutiérrez ${ }^{\mathrm{b}}$ y Luis J. Rodríguez-Muñiz ${ }^{\mathrm{c}}$ \\ a Estudiante de doctorado en la Universidad de Oviedo, Oviedo, Asturias, España \\ ${ }^{\mathrm{b}}$ Departamento de Psicología, Universidad de Oviedo, Oviedo, Asturias, España \\ ${ }^{\mathrm{c}}$ Departamento de Estadística e Investigación Operativa y Didáctica de la Matemática, Universidad de Oviedo, Oviedo, Asturias, España
}

\section{INFORMACIÓN DEL ARTÍCULO}

\section{Historia del artículo:}

Recibido el 3 de septiembre de 2014

Aceptado el 28 de abril de 2015

On-line el 21 de julio de 2015

\section{Palabras clave:}

Educación superior

Universidad

Permanencia

Abandono

\section{R E S U M E N}

El abandono de los estudios superiores es un fenómeno cuya magnitud se ha visto incrementada a la par que el número de estudiantes en la universidad. Supone un importante desaprovechamiento de recursos que trasciende a la persona y cobra especial relevancia en el actual contexto de crisis económica. Por su parte, la implantación del Espacio Europeo de Educación Superior demanda una mejora de resultados educativos que fomenta la implementación de medidas con dicho objetivo. Sin embargo, la escasez de investigaciones sobre abandono desarrolladas en este contexto supone una dificultad añadida, a cuya superación el presente trabajo pretende contribuir.

Se ha desarrollado una investigación bajo un diseño ex post facto para el estudio de la primera cohorte de plena implantación del Espacio Europeo de Educación Superior, ascendiendo la muestra a 1.055 estudiantes.

La información fue recogida mediante la extracción de datos sociodemográficos y de expediente académico de la aplicación de gestión académica de la Universidad de Oviedo, y complementada a través de la aplicación de un cuestionario diseñado ad hoc.

Los resultados subrayan la importancia del rendimiento académico previo, junto con la dedicación del alumno y el tipo de relación establecida con el profesorado, en la decisión final del alumno respecto a su permanencia.

(C) 2014 Instituto de Ciencias de la Educación de la Universidad de Oviedo. Publicado por Elsevier España, S.L.U. Este es un artículo Open Access bajo la licencia CC BY-NC-ND (http://creativecommons.org/licenses/by-nc-nd/4.0/).

\section{Persistence in university studies: The importance of a good start}

\section{A B S T R A C T}

Higher education drop-out is a phenomenon that has been increasing at the same time as the number of students increases in universities. This leads to a significant waste of resources that goes beyond the student and has special relevance in the context of the current economic crisis.

Meanwhile, the implementation of the European Higher Education Area demands an improvement of educational outcomes, which encourages the implementation of measures with that aim. However, the little research had been carried out in this context is an added difficulty. The present work aims to contribute to overcoming this handicap.

An ex post facto design was applied to 1,055 freshman students from the academic year 2010-2011. The information was collected by extracting socio-demographic information and academic records from the academic management software of the University of Oviedo, and supplemented by the application of a survey designed for this purpose.

The results underline the importance of previous academic performance, student engagement, and type of relationship established with faculty staff, on the final decision of the student on whether to continue studies.

(C) 2014 Instituto de Ciencias de la Educación de la Universidad de Oviedo. Published by Elsevier España, S.L.U. This is an open access article under the CC BY-NC-ND license (http://creativecommons.org/licenses/by-nc-nd/4.0/).

\footnotetext{
* Autor para correspondencia. Facultad de Psicología, Despacho 206. Plaza Feijóo, s/n, 33010 Oviedo, España. Tel.: +985109541, 635606765. Correo electrónico: maria_esteban_garcia@hotmail.com (M. Esteban García).
} 


\section{Introducción}

El fenómeno del abandono de los estudios en el nivel universitario ha visto incrementada su magnitud y relevancia a raíz de la democratización del acceso a la educación superior acaecida a lo largo de la segunda mitad del siglo xx. Es por ello que desde entonces, y sobremanera desde los años 80 del siglo pasado, se ha dado una proliferación en cuanto a investigaciones sobre el fenómeno desarrolladas desde diversas perspectivas, que Braxton, Milem y Shaw (2000) han clasificado en 5 enfoques explicativos del abandono en la educación superior, establecidos en función del énfasis otorgado a determinadas variables explicativas, estos son: enfoques psicológico, sociológico, economicista, organizacional e interaccionista.

Sin embargo, y con base en las limitaciones encontradas en la aplicación de enfoques aislados, en la última década surgen una serie de investigaciones que consideran un amplio conjunto de variables implicadas en la manifestación del fenómeno, cuyas interacciones y peso de influencia varían en función de las características del propio sujeto y de su contexto; se trata del enfoque holístico o integrador (Torres, 2010).

Así, las investigaciones desarrolladas bajo un enfoque holístico adoptan una visión humanista y constructivista del ser humano y consideran que los comportamientos de este, como ente complejo, necesitan de un amplio rango de variables explicativas, que darán lugar a diferentes decisiones en función de las particularidades de cada individuo y del contexto en el que se ve inmerso.

Sin embargo, dentro del amplio rango de variables consideradas para la explicación del abandono desde este enfoque destacan algunas identificadas por un amplio número de autores, independientemente del tipo de institución y de la titulación o titulaciones consideradas, estas son: rendimiento académico previo, asistencia a clase, relación con el profesorado y dedicación al estudio. A continuación se da cuenta de algunos trabajos que ilustran la importancia de dichas variables.

En primer lugar, es menester citar por su importancia la investigación longitudinal desarrollada por Montmarquette, Mahseredjian y Houle (2001), en la que recogían información sobre los 3 primeros semestres de universidad de 3.418 participantes procedentes de 43 titulaciones de la Universidad de Montreal. De entre sus resultados destacan la influencia de variables relativas al rendimiento, tanto el rendimiento académico previo como el rendimiento en la universidad, que correlacionan con la permanencia del alumno en la titulación. En el caso de los abandonos más tempranos, tras el primer semestre de universidad, la variable predictiva más influyente es el tamaño de la clase, estando este ligado a la obtención de recursos de apoyo (profesores auxiliares) y obteniendo los mejores resultados las clases medianas de 60-110 estudiantes, donde los alumnos reciben una atención más individualizada.

Los autores también encuentran relación entre el tiempo de dedicación del alumno al trabajo (sea voluntario o remunerado) y el abandono, teniendo mayor probabilidad de abandono aquellos alumnos que mayor tiempo dedican a este tipo de actividades.

Similares resultados son obtenidos por Rodrigo, Molina, GarcíaRos y Pérez-González (2012), que constatan la relación tanto de la nota obtenida en la prueba de acceso a la universidad como de la dedicación a tiempo parcial con el abandono de la titulación.

Tinto (2005), tras la realización de diversas investigaciones, pone de relieve la influencia de las relaciones establecidas por los alumnos (tanto con pares como con personal de la facultad) en el abandono y señala la necesidad de aplicar para la prevención del mismo pedagogías cooperativas y servicios de apoyo complementarios a las actividades de clase.

En esta línea, Braxton et al. (2000) desarrollan una investigación con una muestra de 718 alumnos de nuevo ingreso en la
Universidad de Maryland con el objetivo de explorar la relación entre el aprendizaje activo y la decisión de abandonar o permanecer en la titulación. Sus resultados permiten afirmar que las conductas de clase juegan un rol importante en la toma de estas decisiones.

Por su parte, Arriaga, Burillo, Carpeño y Casaravilla (2011), tras analizar datos relativos a 991 estudiantes de nuevo ingreso en la Universidad Politécnica de Madrid, identifican la diferente influencia de determinadas variables en los diversos tipos de abandono. De sus resultados destacan que aquellos alumnos que permanecen en la titulación inicialmente matriculada manifiestan una mayor dedicación al estudio y un menor compromiso con otras actividades (entre las que cabe mencionar el trabajo remunerado). Así mismo, estos alumnos consideran haber recibido un trato ajustado a sus necesidades por parte del profesorado y cuentan con un mayor grado de experiencias de grupo, en contraposición con lo manifestado por el grupo de alumnos que opta por abandonar la titulación.

De los hallazgos anteriormente expuestos se deduce la importancia que el rendimiento académico previo, la asistencia y participación activa en clase, la cercanía en la relación con el profesorado y la dedicación tanto al estudio como a actividades alternativas tiene para con el fenómeno del abandono. A pesar de que estas investigaciones han supuesto un importante avance del conocimiento científico sobre el problema del abandono y sus posibles medidas preventivas, es preciso reconocer que presentan limitaciones, entre las que destacan el tener una muestra conformada por alumnos de planes anteriores a la implantación del Espacio Europeo de Educación Superior (EEES).

La implantación de un marco común en educación superior a nivel europeo tenía por objetivo el incremento de la competitividad del sistema de enseñanza superior europeo (CE, 1999), así como la movilidad tanto de estudiantes como de trabajadores dentro de la Unión. Los preceptos de calidad y excelencia que impregnan la filosofía del Plan Bolonia deben verse reflejados en sus resultados, y así, las titulaciones han sido diseñadas con base en perfiles profesionales, donde las competencias genéricas, transversales y específicas cobran una especial importancia, cambio que afecta tanto a la didáctica como a la evaluación de las asignaturas de cada titulación (Tobón, Pimienta y García, 2010).

Es por ello que en este nuevo contexto la utilidad del conocimiento producido por los estudios anteriormente comentados ha de ser corroborada, dado que este ha sido obtenido en un contexto educativo sustancialmente diferente al actual y, por tanto, los resultados podrían no ser generalizables. Por ello, el objetivo de la investigación cuyos resultados aquí se presentan era comprobar la influencia de las variables anteriormente mencionadas y que han probado su influencia en el marco de planes de estudio anteriores a la implantación del EEES, en este nuevo contexto.

Con base en los resultados obtenidos por otros investigadores con respecto al fenómeno del abandono, se establecieron las siguientes hipótesis:

H.1. Los estudiantes que permanecen en la titulación inicialmente matriculada manifestarán un rendimiento académico previo a su ingreso en la universidad mayor al de aquellos que la abandonan o cambian de titulación.

H.2. Los estudiantes que permanecen en la titulación asisten a clase en mayor grado que aquellos que la abandonan o cambian de titulación.

H.3. Los estudiantes que permanecen en la titulación manifiestan tener relaciones con el profesorado más cercanas que aquellos que la abandonan o cambian de titulación.

H.4. Los estudiantes que permanecen en la titulación dedican mayor tiempo al estudio que aquellos que optan por cambiar de titulación o abandonar la Universidad de Oviedo. 


\section{Método}

Descripción de los participantes

Los participantes en el estudio que aquí se presenta han sido estudiantes de nuevo ingreso en grado en la Universidad de Oviedo en el curso 2010/11. Dicha población ascendía a 5.215 alumnos, de los que, en septiembre de 2012, 4.194 continuaban estudiando la titulación inicialmente matriculada, 363 habían cambiado de titulación y 658 habían abandonado la Universidad de Oviedo.

Se ha trabajado con una muestra total de 1.055 participantes, formada por 2 submuestras con un nivel de confianza del $97 \%$ y con un margen de error del 4\%: permanecen en la titulación inicialmente matriculada $(\mathrm{N}=626)$ y abandonan esta $(\mathrm{N}=429)$.

Se ha aplicado un procedimiento de muestreo aleatorio estratificado, siendo 5 los estratos, correspondientes a cada una de las ramas de conocimiento. Cabe aclarar que al haberse aplicado un muestreo aleatorio estratificado, todas las titulaciones de cada área de conocimiento han tenido la misma probabilidad de participación en el estudio. La gran diferencia en cuanto a número de alumnos en cada área, tanto en el grupo de los que permanecen como en el de los que cambian de titulación o abandonan la institución, se debe al diferente número de titulaciones disponibles en cada una de ellas (Artes y Humanidades: 9 titulaciones, Ciencias: 7 titulaciones, Ciencias Sociales y Jurídicas: 17 titulaciones e Ingeniería y Arquitectura: 17 titulaciones) y al número de matriculados en cada una de las titulaciones.

Se han establecido cuotas para la submuestra de abandono de titulación en función de si el participante se había matriculado en otra titulación de la Universidad de Oviedo (cambio de titulación, $\mathrm{N}=153$ ) o si, por el contrario, había abandonado esta (abandono, $\mathrm{N}=276$ ).

Así, la distribución final de la muestra se corresponde con la propia de la población estudiada y, por tanto, es representativa de esta (ver tabla 1).

En cuanto a las principales características sociodemográficas de la muestra, el grupo de permanencia manifiesta una edad media de 19,89 años (41,63\% hombres y 58,37\% mujeres), en el grupo de cambio la edad media es de 18,99 años (62,34\% hombres y $37,66 \%$ mujeres), y el grupo de abandono tiene una edad media de 22,18 años (56,16\% hombres y 43,84\% mujeres).

\section{Instrumentos}

Se ha recopilado la información disponible acerca de la población estudiada en la aplicación informática de gestión académica de la universidad:

- Datos de contacto y sociodemográficos: nombre completo, teléfono y correo electrónico, sexo, lugar de nacimiento, nacionalidad y discapacidad (en su caso).

- Datos familiares: familia numerosa, estudios y ocupación de los padres o tutores.

- Datos sobre el acceso a la universidad: nota media de la etapa previa al acceso, nota de acceso, edad de ingreso, fecha de la primera

Tabla 1

Distribución de la muestra $(\mathrm{N}=1055)$

\begin{tabular}{llll}
\hline & Permanece & Cambia & Abandona \\
\hline Arte y humanidades & 62 & 14 & 43 \\
Ciencias & 52 & 12 & 21 \\
Cc. Salud & 83 & 7 & 17 \\
Cc. Soc y jurídicas & 258 & 69 & 111 \\
Ingeniería y Arquitectura & 171 & 51 & 84 \\
Total & 626 & 153 & 276 \\
\hline
\end{tabular}

Fuente: Elaboración propia. matriculación y lugar en el orden de preferencia de los estudios matriculados.

- Información relativa a la titulación matriculada y al desenvolvimiento del estudiante en la misma: rama y titulación matriculada, tipo de centro, disposición de becas, número de créditos matriculados, presentados y aprobados, media del expediente académico y titulación destino del cambio de titulación en su caso.

Dicha información fue complementada con la obtenida mediante un cuestionario diseñado ad hoc para el estudio, cuya aplicación se llevó a cabo a través del correo electrónico o de contacto telefónico. Las variables sobre las que se ha recogido información se refieren a:

- Estado civil, nivel de renta y tipo de residencia durante el curso.

- Motivos de elección tanto de la Universidad de Oviedo como de la titulación matriculada en el curso 2010/11.

- Acciones de acogida universitaria en las que ha participado y valoración del grado de acogida recibido.

- Dedicación al estudio, al trabajo doméstico y a un trabajo remunerado.

- Valoración del esfuerzo requerido por la titulación y satisfacción con las calificaciones.

- Valoración de las relaciones establecidas tanto con los docentes de su titulación como con sus pares.

- Planteamiento de la posibilidad de abandonar la titulación y motivos, en su caso.

- En caso de interrupción o abandono de los estudios, situación actual y grado de satisfacción con dicho cambio.

- Satisfacción general con la Universidad de Oviedo (grado en que la recomendarían).

Los ítems empleados para la recogida de información podían ser de 3 tipos:

- Preguntas cerradas con determinado número de alternativas de respuesta (entre 3 y 8 ).

- Preguntas cerradas en formato de escala tipo Likert.

- Preguntas abiertas, empleadas únicamente para cuantificar el número de horas que el participante empleaba en el desarrollo de una determinada actividad.

A continuación se da cuenta de la operativización de aquellas variables, cuyo estudio resume el presente artículo:

- Grupo: la variable "grupo» se refiere al grupo al que pertenece el alumno según su situación académica en el momento del desarrollo de la investigación (permanece en la titulación inicialmente matriculada, ha cambiado de titulación aunque permanece en la Universidad de Oviedo o ha abandonado los estudios en la Universidad de Oviedo).

- Rendimiento académico previo: a fin de valorar el rendimiento académico previo al ingreso en la universidad de forma homogénea en todos los participantes se ha operativizado esta variable como la nota media obtenida en Bachillerato o Ciclo Formativo y la nota en la fase general de la Prueba de Acceso a la Universidad. - Asistencia a clase y valoración del grado de cercanía en la relación con el profesorado: la operativización de estas variables se ha concretado en ítems tipo escala Likert. En el primer caso, se solicitó a los participantes que valorasen su grado habitual de asistencia a clase, pudiendo ser la respuesta "nada», "poco», "regular», «bastante» o "mucho». En el segundo caso, se solicitó a los participantes que valorasen de manera general su relación con los profesores de la titulación, pudiendo ser la respuesta «nada cercana», "poco cercana», "regular», «bastante cercana» o «muy cercana». 
- Dedicación al estudio: se ha pedido a los participantes que expresasen su número medio de horas semanales dedicadas al estudio, así como el dedicado a otras actividades laborales no vinculadas al estudio que pueden interferir en este (Rué, 2014).

\section{Procedimiento}

Se ha trabajado con base en un diseño de investigación ex post facto, explicativo y correlacional. Diseños similares han sido anteriormente aplicados por Araque, Roldán y Salguero (2009) o Villacorta (2010).

Dicho diseño ha sido desarrollado desde un enfoque holístico, considerando esta opción la más adecuada, dadas las características de nuestra institución y el carácter cultural y contextual del fenómeno analizado.

La recogida de información se ha llevado a cabo mediante 2 procedimientos:

- Solicitud de datos personales y expediente académico de los alumnos.

- Aplicación de encuesta online o telefónica asistida por ordenador (computer-assisted telephone interviewing).

Los análisis descriptivos y correlacionales realizados sobre la información recabada se han llevado a cabo con el programa R Development Core Team (versión 2.15, disponible en http://www.R-project.org). Se ha empleado el valor $p$ para el contraste de la hipótesis nula en todos los casos, siendo este el menor nivel de significación al cual puede rechazarse la hipótesis nula.

\section{Resultados}

A continuación se presentan los resultados obtenidos en la investigación, explorando la posible relación de la variable grupo con el resto, siendo esta el factor de comparación entre grupos empleado. Como anteriormente se ha comentado, dicha variable se ha configurado en función de los datos disponibles en el servicio de matriculación a principios del curso 2012/13, pudiendo esta adoptar 3 valores, a saber: permanece, cambia de titulación y abandona la Universidad de Oviedo.

Es necesario aclarar aquí que se ha aplicado la definición de abandono más ampliamente aceptada, entendiendo por estudiante que abandona aquel que habiendo iniciado una determinada titulación, y antes de finalizarla, no se matricula en la misma por 2 cursos consecutivos (Cabrera et al., 2006a,bCabrera, Tomás, Álvarez y González, 2006), pudiendo optar por un cambio de titulación o por el abandono de la institución que le dio acogida.

Así pues, los análisis realizados permiten localizar las principales diferencias entre los 3 colectivos mencionados, así como servir de base a intervenciones que contribuyan a mejorar la permanencia en nuestra universidad. La tabla 2 resume los principales estadísticos descriptivos relativos a las mencionadas variables, mostrándose a continuación los resultados relativos a la relación de cada una de estas con el fenómeno estudiado.

\section{Rendimiento académico previo al ingreso en la universidad}

En cuanto a si se da relación del rendimiento previo al ingreso en la universidad con el fenómeno estudiado, es preciso afirmar que se obtienen diferentes resultados según la nota de referencia. En el caso de la nota media en Bachillerato o Ciclos Formativos se encuentran diferencias estadísticamente significativas en función del grupo al que se adscribe el alumno según su situación académica, pero solamente entre los grupos abandona y permanece $(p=.000 ; F=47,55)$, presentando calificaciones más bajas aquellos alumnos que abandonan la institución respecto a los que permanecen o cambian de titulación.

En el caso de la nota obtenida en la fase general de la Prueba de Acceso a la Universidad, se ha confirmado también la relación de esta variable con el fenómeno estudiado para los 3 grupos $(p=.000$; $F=27,50$ ), presentando las notas más altas los alumnos que permanecen, seguidos por los que cambian de titulación y habiendo obtenido las puntuaciones más bajas aquellos que abandonan la institución donde iniciaron sus estudios universitarios.

\section{Asistencia a clase}

Se ha encontrado relación entre las variables "grupo" y "grado de asistencia a clase» ( $p=.000 ; F=83,32$ ), dándose una tendencia a asistir a clase en grado nada, poco o regular para los grupos de alumnos de abandono y cambio, y la tendencia inversa para el grupo de aquellos alumnos que permanecen.

\section{Valoración del grado de cercanía en la relación con el profesorado}

Como se ha comentado con anterioridad, una relación de confianza entre alumnado y profesorado favorece un adecuado clima de estudio, al tiempo que mejora los resultados educativos (Castro, 2012). Es por ello que se ha explorado el comportamiento de esta variable para cada uno de los grupos establecidos; encontramos valores muy parecidos entre un grupo y otro, muy cercanos todos al valor 3 (regular) y cuyos estadísticos descriptivos pueden ser consultados en la tabla 2. Sin embargo, se ha confirmado la relación entre dicha variable y el problema del abandono, tomando como factor comparativo la variable "grupo» $(p=.000 ; F=8,31)$, dándose una tendencia a considerar las relaciones con el profesorado poco o nada cercanas en los grupos de abandono y cambio, y manifestando una distribución gaussiana el grupo de alumnos que permanece.

\section{Tabla 2}

Estadísticos descriptivos relativos a las variables estudiadas

\begin{tabular}{|c|c|c|c|c|c|c|c|c|}
\hline & \multicolumn{2}{|c|}{ Permanece } & \multicolumn{2}{|c|}{ Cambia } & \multicolumn{2}{|c|}{ Abandona } & \multirow[t]{2}{*}{ Asimetría } & \multirow[t]{2}{*}{ Curtosis } \\
\hline & $\bar{X}$ & DT & $\bar{X}$ & DT & $\bar{X}$ & DT & & \\
\hline Rendimiento Bachillerato/Ciclo Formativo & 7,39 & 1,32 & 6,57 & 1,05 & 6,48 & 0,89 & 0,66 & $-0,55$ \\
\hline Rendimiento fase general PAU & 6,81 & 1,37 & 6,26 & 1,18 & 5,92 & 1,11 & 0,21 & $-0,76$ \\
\hline Asistencia a clase & 4,47 & 0,78 & 4,08 & 0,98 & 3,63 & 1,1 & $-1,13$ & 0,62 \\
\hline Cercanía en la relación con profesorado & 3,45 & 0,9 & 3,3 & 0,96 & 3,18 & 1,04 & 0,51 & 0,00 \\
\hline Horas semanales de estudio & 13,02 & 9,09 & 11,01 & 6,94 & 9,34 & 6,29 & 1,47 & 3,32 \\
\hline Horas semanales de trabajo doméstico & 5,05 & 6,5 & 4,49 & 4,60 & 5,11 & 6,30 & 4,7 & 38,27 \\
\hline Horas semanales de trabajo remunerado & 3,59 & 9,45 & 2,29 & 6,31 & 5,97 & 12,56 & 3,02 & 9,61 \\
\hline
\end{tabular}

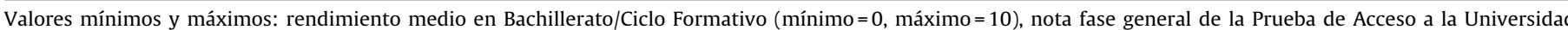

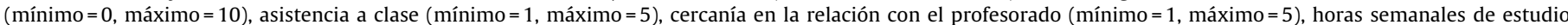
$($ mínimo $=0$, máximo $=60$ ), horas semanales de trabajo doméstico (mínimo $=0$, máximo $=70$ ) y horas semanales de trabajo remunerado $($ mínimo $=0$, máximo $=81$ ).

Fuente: Elaboración propia. 


\section{Dedicación al estudio}

Se ha considerado oportuno explorar la dedicación al estudio de los participantes en la investigación en términos de horas semanales promedio dedicadas a esta actividad. Sin embargo, al tratar de explorar la posible relación de esta variable con el grupo al que se adscribe el sujeto según su situación académica, la dispersión de los datos ha propiciado que no se pudieran obtener resultados estadísticamente significativos en este sentido. Por ello, se ha optado por una medida indirecta (horas semanales promedio de trabajo doméstico y trabajo remunerado), entendiendo que una alta dedicación a estas otras actividades resta dedicación a las propias actividades de estudio y favorece el abandono (Rué, 2014).

Los datos presentados en la tabla 2 (media y desviación típica) son una muestra de que aunque para la mayoría de los estudiantes el tiempo dedicado al trabajo doméstico y al trabajo remunerado es escaso, existe una minoría que dedica un tiempo mucho mayor a estas actividades, pudiendo interferir en su desenvolvimiento académico.

En lo referente a la posible relación entre el fenómeno estudiado y la dedicación que el estudiante manifiesta para con el trabajo doméstico y para con un trabajo remunerado, en el primer caso no se han encontrado diferencias estadísticamente significativas suficientes para poder afirmar la existencia de tal relación $(p=.56$; $F=0,57$ ). Sin embargo, en el segundo caso («horas semanales de trabajo remunerado») sí se ha encontrado significación estadística $(p=.000 ; F=8,11)$.

Así, podemos afirmar que el compromiso académico del estudiante y el riesgo de abandonar los estudios que cursa están relacionados con la dedicación que este manifiesta respecto a otras actividades de las que es responsable, entre las que cabe destacar el trabajo remunerado. Es por ello que sería recomendable diseñar medidas que favorezcan la conciliación de la vida académica con la vida laboral y familiar.

\section{Conclusiones}

El objetivo planteado para la investigación cuyos resultados aquí se han presentado era comprobar la consistencia de los resultados obtenidos por otros investigadores sobre determinadas variables influyentes en el fenómeno del abandono, dado el cambio de contexto acaecido a tenor de la implantación del EEES.

Los resultados anteriormente expuestos responden al objetivo que el equipo de investigación se había planteado, siendo coherentes con los obtenidos por diversos investigadores respecto a estudios no adaptados al EEES, lo que informa de su consistencia y permite utilizarlos como base para el establecimiento de acciones correctivas:

El rendimiento académico previo al ingreso en la universidad es una variable que ha confirmado su influencia en la permanencia del alumno universitario (Antoni, 2003; Araque et al., 2009; Belloc, Maruotti y Petrella, 2010; Di Pietro, 2006; Rodríguez, 2011; Smith y Naylor, 2001). Algunos autores, entre los que destaca Broc (2011), han constatado que esta variable es la que mayor correlación presenta con el rendimiento académico final. Por ello, es recomendable el establecimiento de cursos 0 y cursos de técnicas de estudio para aquellos alumnos cuyo rendimiento previo se sitúe por debajo de la media en los estudios destino o procedan de itinerarios formativos escasamente relacionados con la titulación matriculada.

Por su parte, la variable asistencia a clase ha sido tradicionalmente considerada una variable facilitadora de un óptimo rendimiento académico. Martínez y Álvarez (2005), p. 130) explican esta influencia afirmando que "necesariamente, un alumno que falta reiteradas veces al centro verá mermado su aprovechamiento o rendimiento educativo por desconectarse del ritmo habitual de las clases, de la secuencia de las explicaciones, actividades, evaluaciones e interacciones en el aula».

En la presente investigación, se ha confirmado la relación entre el fenómeno del abandono y el grado de asistencia a clase, teniendo mayores probabilidades de permanencia aquellos alumnos que asisten a clase con mayor frecuencia. Estos resultados coinciden con los obtenidos por Cabrera et al. (2006)Cabrera, Bethencourt, González y Álvarez (2006) y Casaravilla, del Campo, García y Torralba (2012), que incluso han llegado a calificarla como variable predictora del abandono de titulación.

Con la introducción del EEES, la asistencia a clase cobra aún mayor relevancia en el ámbito universitario: al concebir la enseñanza desde una perspectiva de formación integral, otorga gran importancia a los procesos de orientación de alumnos, sobremanera a los distintos tipos de tutoría universitaria que se desarrollan en el contexto del aula (tutoría de asignatura y tutoría de titulación). En este sentido, las universidades han instaurado diferentes medidas para promocionar la función tutorial, entre las que destaca el Plan Universitario de Tutoría de la Facultad de Educación de la Universidad de La Laguna, cuyos resultados han sido evaluados por Álvarez (2012), que concluye afirmando que dicho instrumento aumenta la responsabilidad de los participantes para con su futuro y contribuye a prevenir el abandono.

En relación con las funciones docentes y orientadoras que ha de desarrollar el profesorado universitario en el marco del EEES, está el grado de cercanía establecido en las relaciones profesor-alumno. Su influencia en el fenómeno estudiado ha quedado constatada, manifestando los grupos de cambio y abandono una tendencia a considerar las relaciones con su profesorado poco o nada cercanas. Si bien esta variable no puede ser considerada como determinante del abandono de los estudios, sí resulta de influencia en el fenómeno en la medida en que contribuye al establecimiento de un adecuado clima escolar, tal y como señala Castro (2012). Esta autora va más allá, y con base en una profunda revisión bibliográfica sobre el tema, afirma que «muchos de los problemas educativos existentes se refieren a cuestiones no estrictamente relacionadas con los contenidos, sino con aspectos y factores contextuales, organizativos y de relaciones personales, que inciden en los resultados educativos finales» (Castro, 2012, p. 35).

Por ello, resulta conveniente la mejora de la formación inicial y permanente del profesorado universitario, la potenciación de las tutorías (tanto de asignatura como de carrera) y el establecimiento de mecanismos de coordinación entre servicios y departamentos universitarios, a fin de dotar de mayor calidad tanto al sistema de información y orientación al estudiante como a la propia docencia universitaria y el clima escolar, proporcionando un mejor soporte a los estudiantes y transformando la universidad en un escenario de salud, en la línea de lo planteado por Prior et al. (2011) a tenor de las propuestas de la Organización Mundial de la Salud.

Tanto la asistencia a clase como la dedicación al estudio por parte del alumno universitario pueden verse reducidas a tenor del tiempo que este dedica a otras tareas de las que es responsable. En este sentido, se ha constatado la relación entre el tiempo dedicado a un trabajo remunerado y el abandono de titulación, resultados que concuerdan con los encontrados por Corominas (2001), Montmarquette et al. (2001) y Rué (2014), entre otros autores. En este sentido, Jiménez y Márquez (2014), p. 7), en el marco de una investigación sobre dificultades y factores facilitadores para cursar estudios universitarios, señalan que "los problemas para compatibilizar estudios, trabajo y familia adquieren especial significación para el grupo mayor de 30 años, y en particular, entre las mujeres»; se trata de la denominada problemática del estudiante adulto, perfil de estudiante cada vez más presente en las universidades según los análisis de estadísticas nacionales realizados por Cruz, Learreta, Huertas, Rodriguez y Ruiz (2011). Es por ello recomendable el establecimiento de medidas que favorezcan la compatibilización de 
estudios y empleo, entre las que cabe destacar el ofrecer horarios que favorezcan la conciliación de ambos (Casaravilla, 2014).

Una vez discutidos los resultados obtenidos, es momento para comentar la posible confirmación de las hipótesis de partida; estas establecían que los estudiantes que permanecen en la titulación inicialmente matriculada presentarían características diferenciales respecto a aquellos estudiantes que la abandonan en cuanto a su rendimiento académico previo (H.1), asistencia a clase (H.2), cercanía en la relación con el profesorado (H.3) y dedicación al estudio (H.4). Tras revisar los resultados obtenidos es posible afirmar que tan solo la segunda y la tercera hipótesis se cumplen totalmente, puesto que los resultados ponen de relieve que los grupos de cambio y abandono presentan diferencias significativas respecto al grupo que permanece en dichas variables. Sin embargo, la H.1 no se cumple totalmente, ya que los alumnos que permanecen tan solo presentan diferencias significativas respecto a los otros grupos en cuanto a rendimiento previo en términos de nota en la fase general de la Prueba de Acceso a la Universidad.

En cuanto a la H.4, que afirmaba que aquellos alumnos que permanecían dedicarían un mayor tiempo al estudio que aquellos otros que optan por abandonar, es preciso reconocer que la dispersión de los datos ha imposibilitado la comprobación de manera directa. Sin embargo, al tomar medidas indirectas de esta variable (compromiso con otras actividades que pueden mermar la dedicación al estudio) sí se ha podido confirmar la relación, y así, los estudiantes que abandonan o cambian de titulación dedican mayor tiempo a un trabajo remunerado que aquellos que permanecen.

Por último, es menester identificar como principal limitación de la investigación aquí presentada que los resultados aquí expuestos caracterizan el abandono de titulación en primer curso de carrera, ya que se ha asumido la definición de abandono más ampliamente aceptada (estudiante que habiendo iniciado determinados estudios, y antes de finalizarlos, no se matricula en los mismos por 2 cursos consecutivos). Por ello, a pesar de que «las referencias sugieren una concentración sustantiva del [abandono] en el mismo año de transición a la educación universitaria» (Villar, Vieira, Hernández y Almeida, 2012, p. 151), es conveniente realizar un seguimiento de la cohorte para evaluar si el abandono en segundo y posteriores cursos -aunque de menor magnitud que el abandono en primeropresenta particularidades respecto a este.

\section{Conflicto de intereses}

Los autores declaran no tener ningún conflicto de intereses.

\section{Financiación}

Investigación financiada por parte del Ministerio de Educación, Cultura y Deporte del PRoyecto Integral de ORientación AcadémicoProfesional de la Universidad de Oviedo (PRIOR).

\section{Agradecimientos}

Se agradece a Ignacio Grana, COIE y Servicio de Informática de la Universidad de Oviedo su colaboración para la obtención de los datos necesarios para el desarrollo de la investigación que sustenta este artículo.

\section{Referencias bibliográficas}

Álvarez, P. R. (2012). Los planes institucionales de tutoría y el desarrollo de competencias en el marco del Espacio Europeo de Educación Superior. Perfiles Educativos, 34(137), 28-45

Antoni, E. J. (2003). Alumnos universitarios: el porqué de sus éxitos y fracasos. Buenos Aires: Miño y Dávila Editores.

Araque, F., Roldán, C. y Salguero, A. (2009). Factors influencing university drop out rates. Computers E'Education, 53, 563-574, org/10.1016/j.compedu.2009.03.013

Arriaga, J., Burillo, V., Carpeño, A. y Casaravilla, A. (2011). Caracterización de los tipos de abandono. Dividamos el problema y venceremos más fácilmente.
I Conferencia Latinoamericana sobre el Abandono en la Enseñanza Superior Managua, Nicaragua., 17 y 18 de noviembre [consultado 13 Jul 2012].[consultado 13 Jul 2012] Disponible en: http://www.clabes2011-alfaguia.org.pa/ponencias ST_1_Abandono/7_JArriaga_Perfiles\%20abandono.pdf.

Belloc, F., Maruotti, A. y Petrella, L. (2011). How individual characteristics affect university students drop-out: A semiparametric mixed-effects mode for an Italian case study. Journal of Applied Statistics, 38(10), 2225-2239. http://dx.doi.org/10.1080/02664763.2010.545373

Braxton, J. M., Milem, J. M. y Shaw, A. (2000). The influence of active learning on the college student departure process: Toward a revision of Tinto's theory. The Journal of Higher Education, 71(5), 569-590.

Broc, M. A. (2011). Voluntad para estudiar, regulación del esfuerzo, gestión eficaz del tiempo y rendimiento académico en alumnos universitarios. Revista de Investigación Educativa, 29(1), 171-185.

Cabrera, L., Bethencourt, J. T., González, M. y Álvarez, P. (2006). Un estudio transversal retrospectivo sobre la prolongación y abandono de estudios universitarios. Revista Electrónica de Investigación y Evaluación Educativa, 12(1), 105-127.

Cabrera, L., Tomás, J., Álvarez, P. y González, M. (2006). El problema del abandono de los estudios universitarios. Revista Electrónica de Investigación y Evaluación Educativa, 12(2), 171-203, http://www.uv.es/RELIEVE/v12n2/RELIEVEv12n2_1.htm.

Casaravilla, A. (2014). El abandono académico: análisis y propuestas paliativas. Dos proyectos de la Universidad Politécnica de Madrid. Revista Pensamiento Matemático, 4(1), 7-15.

Casaravilla, A., del Campo, J. M., García, A. y Torralba, M. R. (2012). Análisis del abandono de los estudios de ingeniería y arquitectura en la Universidad Politécnica de Madrid. II Conferencia Latinoamericana sobre el Abandono de la Enseñanza Superior. Porto Alegre, Brasil, 8 y9 de noviembre.

Castro, S. M. (2012). Tipos de aula y ambiente social en el proceso de aprendizaje en el nivel de educación básica de la ciudad de Salinas, año 2012. Universidad Técnica Particular de Loja, Ecuador [consultado 25 May 2015]. Disponible en: http://dspace.utpl.edu.ec//handle/123456789/3547

CE (1999). El Espacio Europeo de la Enseñanza Superior. Declaración conjunta de los ministros europeos de educación reunidos en Bolonia el 19 de junio de 1999. Universidad de Castilla-La Mancha [consultado 25 May 2015] Disponible en: http://www.ond.vlaanderen.be/hogeronderwijs/bologna/links/language/1999 Bologna_Declaration_Spanish.pdf

Corominas, E. (2001). La transición a los estudios universitarios. Abandono o cambio en el primer año de universidad. Revista de Investigación Educativa, 19(1), $127-151$

Cruz, A., Learreta, B., Huertas, P., Rodriguez, B. y Ruiz, M. (2011). Estudiantes adultos matriculados en la universidad española. (Universidad Europea de Madrid) [consultado 2 Ene 2015]. Disponible en: http://www.dsw.edu.pl/fileadmin/wwwranlhe/files/Cruz_et-al.pdf

Di Pietro, G. (2006). Regional labour market conditions and university dropout rates Evidence from Italy. Regional Studies, 40(6), 617-630.

Jiménez, M. L. y Márquez, E. (2014). Ir a la universidad después de los 30: dificultades y factores facilitadores. Aula Abierta, 42, 1-8.

Martínez, R. A. y Álvarez, L. (2005). Fracaso y abandono escolar en Educación Secundaria Obligatoria: implicación de la familia y los centros escolares. Aula Abierta, 85, 127-146.

Montmarquette, C., Mahseredjian, S. y Houle, R. (2001). The determinants of university dropouts: A bivariate probability model with sample selection. Economics of Education Review, 20, 475-484.

Prior, M., Manzano, E., Villar, E., Caparrós, B., Juan, J.y Luz, E. (2011). Estilos comunicativos, vinculación universitaria y adaptación psicosocial. Revista de Investigación Educativa, 29(2), 387-405.

Rodrigo, M. F., Molina, J. G., García-Ros, R. y Pérez-González, F. (2012). Efectos de interacción en la predicción del abandono en los estudios de Psicología. Anales de Psicología, 28(1), 113-119.

Rodríguez, J. A. (2011). Fracaso académico en la universidad: un estudio piloto a través de la complementación metodológica para el análisis de sus posibles causas. Tesis Doctoral. Universidad Jaume I, Castellón, España.

Rué, J. (2014). El abandono universitario: variables, marcos de referencia y políticas de calidad. Revista de Docencia Universitaria, 12(2), 281-306.

Smith, J. P. y Naylor, R. A. (2001). Dropping out of university: A statistical analysis of the probability of withdrawal for U. K. university students. Journal of Royal Statistical Society-Series A, 164, 389-405.

Tinto, V. (2005). Student retention: What next? National Conference on Student Recruitment, Marketing, and Retention. Washington, D. C., U. S. A., July 27-30 [consultado 25 May 2015]. Disponible en: http://www.gvsu.edu/cms3/assets B85DAC41-B7B8-3B9F-A116121D5AE29B05/Student\%20Retention-What\%20 Next.pdf

Tobón, S., Pimienta, J. H. y García, J. A. (2010). Secuencias didácticas: aprendizaje y evaluación de competencias. Madrid: Pearson.

Torres, L. E. (2010). Estado del arte de la retención de estudiantes de la educación superior. Colombia: Pontificia Universidad Javeriana. Bogotá D. C. [consultado 25 May 2015]. Disponible en: http://www.javeriana.edu.co/documents/ 15838/273636/Retenci\%C3\%B3nEstudiantil2012.pdf/124fdba5-2318-432a-8e9f $-126 a 2501 c 229$

Villacorta, E. (2010). Inteligencia emocional y rendimiento académico en estudiantes de Medicina Humana de la Universidad Nacional de la Amazonía Peruana. Ciencic y Desarrollo, 12, 41-56.

Villar, A., Vieira, M. M., Hernández, F. J. y Nunes, A. (2012). Más que abandono de los estudios, trayectorias de reubicación universitaria. Revista Lusófona de Educação, $21,139-216$. 